\title{
Improvement of Tuning Fork Gyroscope Drive-mode Oscillation Matched using a Differential Driving Suspension Frame
}

\author{
Thang Nguyen Van', Tran-Duc Tan ${ }^{2}$, Hung Vu Ngoc ${ }^{3}$, Trinh Chu Duc ${ }^{4}$ \\ ${ }^{1}$ Broadcasting College I, Radio the voice of Vietnam \\ ${ }^{2,4}$ University of Engineering and Technology, Vietnam National University, Hanoi, Vietnam \\ ${ }^{3}$ University of Science and Technology, Hanoi, Vietnam
}

\begin{tabular}{l}
\hline \hline Article Info \\
\hline Article history: \\
Received Jun 5, 2016 \\
Revised Sep 30, 2016 \\
Accepted Oct 13, 2016 \\
\hline
\end{tabular}

\section{Keyword:}

Differential driving suspension coupling spring

Differential MEMS gyroscope

Electronics differential

amplifier

Vibration tuning fork gyroscope

\begin{abstract}
This paper presents a novel design of a vibration tuning fork gyroscope (TFG) based on a differential driving suspension coupling spring between two gyroscopes. The proposed TFG is equivalent to a transistor differential amplifier circuit. The mechanical vibrations of driving frames are, therefore, well matched. The matching level depends on stiffness of spring. When three various TFG structures respond to differential stiffness of spring, their the driving frame mechanical vibration is well matched in case the input excitation driving differential phase is less than $3.5^{\circ}, 2.5^{\circ}$, and $4^{\circ}$, respectively. The fabricated tuning fork gyroscope linearly operates in the range from -200 to +200 degree/s with the resolution of about $0.45 \mathrm{mV} /$ degree $/ \mathrm{s}$.
\end{abstract}

Copyright $\odot 2016$ Institute of Advanced Engineering and Science. All rights reserved.

Corresponding Author:

Thang Nguyen Van,

Faculty of Electronics and Communications Technology,

Broadcasting College 1,

136 Quy Luu Road, Phu Ly City, Ha Nam Province, Vietnam.

Email: nguyenbathangvov@gmail.com

\section{INTRODUCTION}

Nowadays, micromachined gyroscope in general and vibratory tuning fork gyroscope in particular are very popularly utilized in reality [1-4]. In many applications, performance and operation of gyroscope and TFGs are affected by a wide variety of changing environmental conditions such as pressure, ambient vibrations, and temperature [5-7]. The robustness of sensors and beams to these external influences during operation is critical for adequate performance [8].

All micromachined vibratory gyroscopes can be divided into two various types: Type I and Type II [9]. The working principle of all of them is based on the Coriolis force produced by rotation of the gyroscope causing a transfer of energy between two of the gyroscope' modes of vibration. A new MEMS gyroscope design can improve performance of angle measurement implemented in [10] while the other is developed to either upgrade the performance or reduce the cost [11]. Besides that, there are also some other optimal MEMS gyroscope structures such as micromachined ring or disk designed to obtain the better precision [12-13]. A detailed analysis of the cause of vibration-induced error is implemented to understand the vibration effects on ideal tuning fork gyroscopes [5]. The article points out three major causes of error that arise from capacitive nonlinearity at the sense electrode, asymmetric electrostatic forces along sense direction at the drive electrodes and asymmetric electrostatic forces along drive direction at the drive electrodes. Reference [14] utilizes symmetrically decoupled tines with drive-mode synchronization and sense-mode coupling structures. The levered drive-mode mechanism structurally forces the anti-parallel, antiphase drive-mode motion and eliminates the lower frequency spurious mode presented in conventional 
tuning fork gyroscopes. The linearly coupled, momentum and torque balanced anti-phase sense-mode reduces dissipation of energy through the substrate yielding ultra-high quality factors. A completely symmetric, dynamically balanced quadruple mass gyroscope with a $2.2 \mathrm{kHz}$ operational frequency illustrated virtually identical drive and sense mode Q-factors of 0.9 million. The most important thing in the quadruple mass design is to be expected to enable rate-integrating mode of operation due to its unique combination of low energy dissipation and isotropy of both the resonant frequency and damping [15]. A novel micromachined dual-axis TFG could effectively minimize the undesired lateral motion and ensure the anti-phase resonant mode of the two vibration frames [16]. A capacitive-type tuning fork micro-gyroscope is developed in [17]. A novel quad mass gyroscope in [18-19] is designed and fabricated in order to increase the gap between two resonance modes and reduce the energy transfer between the two modes, allowing for robustness to external acceleration.

However, there are not any studies mentioning and analyzing about the importance and the role of differential driving suspension coupling spring and suspension beams between two gyroscopes in conventional tuning fork gyroscope.

This paper presents a novel design of a vibration tuning fork gyroscope based on a differential driving suspension coupling spring between the two driving frames of TFGs. Therefore, the mechanical vibrations of driving frames are well matched. It means that the differential driving suspension coupling spring can compensate differential phase shift of two input excitation driving signals allowing the TFG to work normally.

\section{TUNING FORK GYROSCOPE AND DIFFERENTIAL AMPLIER}

\subsection{Gyroscope}

A 2-DOF vibratory rate gyroscope is shown in figure 1 . In fact, it is comprised of a proof mass suspended above the substrate and the proof-mass is supported by anchored flexures which play the role of a flexible suspension between the substrate and the proof-mass, allowing the proof-mass free to oscillate in two orthogonal directions (the drive and sense directions). In the drive mode, the suspension system allows the proof-mass to oscillate in the drive direction. The proof-mass is driven into resonance in the drive direction by an external sinusoidal force at resonant frequency of the drive mode. The sense mode accelerometer is formed by the proof-mass. The system of suspension allows the proof-mass to oscillate in the sense direction. In case gyroscope is subjected to an angular rotation, a sinusoidal Coriolis force at the frequency of drive mode oscillation is induced in the sense direction. The Coriolis force excites the sense mode accelerometer, causing the proof-mass to respond in the sense direction [8].

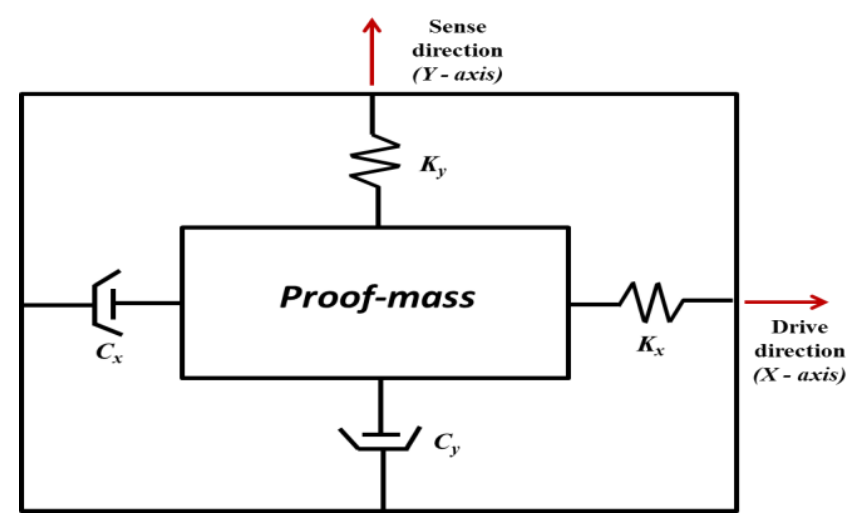

Figure 1. A 2-DOF Vibratory Rate Gyroscope

\subsection{Vibration Rate Tuning Fork Gyroscope Model}

The single mass vibratory rate gyroscope with conventional drive and sense mode oscillations is very sensitive to variations in system parameters that shift the drive or sense resonant frequencies [8]. Instead of single mass, TFG uses a pair of masses driven to resonance. TFG realizes the Coriolis acceleration to improve the sensitivity by using a differential suspended masses structure.

Working principle of the TFG can be compared with the electronics differential amplifier based on two transistors and a current source, see Figure 2. 


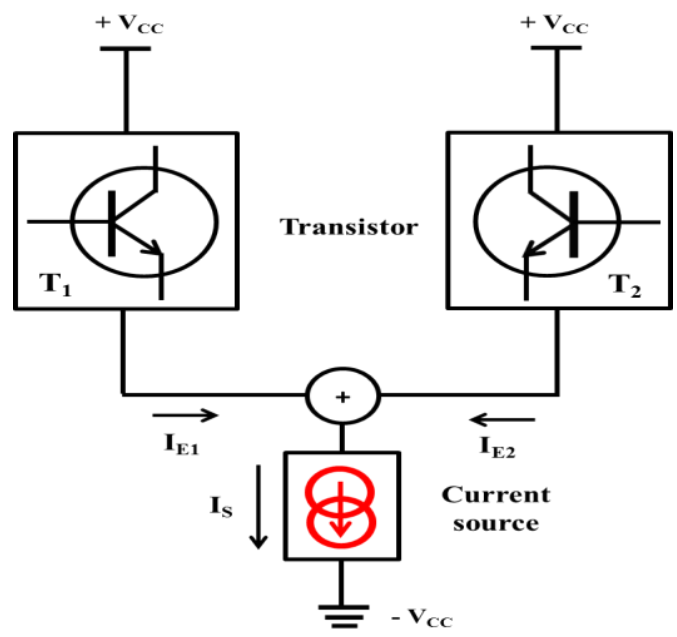

Figure 2. The Electronics Differential Amplifier Basing on Two Transistors and a Current Source

In Figure 2, the differential pair amplifier is formed from the same name two transistors, where their emitters are connected together. The shared emitter node is fed from a constant current source. The two base inputs can be applied a differential input signal and the two outputs from the collectors remain balanced.

As a transistor amplifies the current flowing between base and emitter, it follows that the current flowing in the collector circuit of the first transistor is proportional to the difference between the two inputs. However, since the circuit is totally symmetrical, the element can be viewed either as an amplifier or as an emitter follower, understanding does not depend on which role you assign to which device. The commonmode rejection ratio (CMRR) of the differential amplifier is linearly related on the current source resistance Re [20], whose value is ideally infinite. Therefore, in general, the CMRR parameter of a differential amplifier is extremely large in comparing with a single stage amplifier. The CMRR is up to hundred thousand or even more in some cases. Hence, the differential signal between the two inputs is much amplified and the common signal is rejected.

Based on the working principle of the electronic differential amplifier, a TFG with differential suspended driving masses is proposed (see Figure 3).

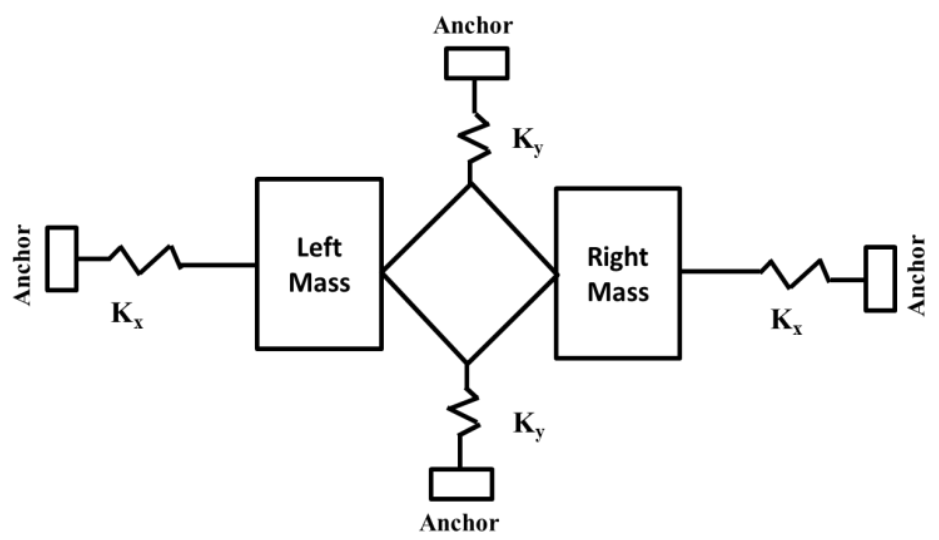

Figure 3. TFG with two differential suspended driving masses

In this structure, two driving masses are hanged on $K_{x}$ springs. They allow driving masses to oscillate only on the $x$-axis. The diamond frame with two $K_{y}$ springs play a role as the current source on the electronic amplifier. By using this diamond suspension, the two driving masses oscillate with same amplitude but phase reversal. The proposed TFG, therefore, improves the sensitivity and much rejects the commonmode noise from both driving and sensing sections. 


\section{DIFFERENTIAL DRIVING OSCILLATED TFG DESIGN}

Figure 4 shows 2-DOF design of a proposed single gyroscope. The driving frame is suspended on the $x$-axis springs. Capacitive actuator drives the frame to oscillate on driving resonant frequency. The sensing mass is hanged on the driving frame by using two $y$-axis springs. In case of having excited driving signals, the driving frame oscillates along the $x$-axis. When gyroscope is effected by $\omega$ angular rate, the sensing frame oscillates along the $y$-axis thanks to two ellipse shape $y$-axis springs.

The driving oscillation is excited by applying a voltage to capacitor pairs. In the case of driving function simulation, a mechanic force can be directly applied to drive comb frames.

In this work, the designed parameters of the single gyroscope are shown in the table 1 . Several eigenfrequencies are listed in the Table 2. The first oscillation mode with frequency of $13544.7 \mathrm{~Hz}$ is the driving resonant oscillation one. Therefore, the driving proof-mass always obtains the maximum amplitude displacement when applying this resonant frequency as the excited signal. The rest modes in the Table 2 are unwanted driving and sensing oscillations. Mass of driving proof-mass (including of $0.9408 \times 10 \mathrm{e}-11 \mathrm{~kg}$ sensing proof-mass) is $0.5452 \times 10 \mathrm{e}-7 \mathrm{~kg}$. The stiffness of drive mode springs $K_{d}=347 \mathrm{~N} / \mathrm{m}$ (including eights springs) and the stiffness of sense mode springs $K_{s}=540 \mathrm{~N} / \mathrm{m}$ (including two ellipse shape springs).

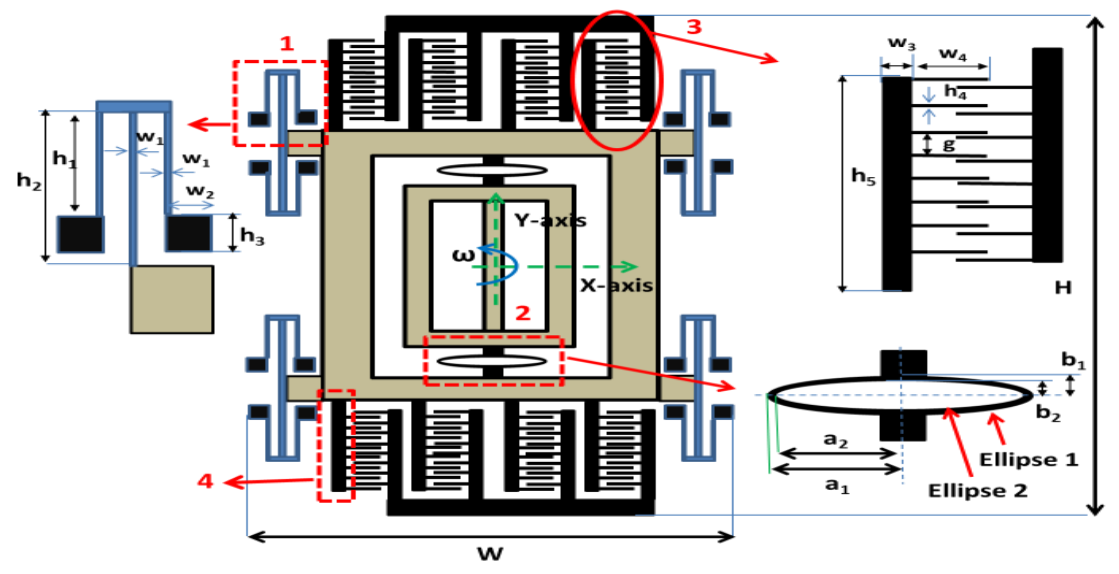

Figure 4. The Proposed Gyroscope: (1) Drive Springs, (2) Sense Springs, (3) Drive Capacitor Pairs, (4) Drive Comb Frame

Table 1. The Proposed Gyroscope Structure Parameters

\begin{tabular}{|c|c|c|}
\hline Parameter & Symbol & Value \\
\hline Real gyroscope height & $H$ & $1754 \mu \mathrm{m}$ \\
\hline Real gyroscope width & $W$ & $1644 \mu \mathrm{m}$ \\
\hline Device thickness & $t$ & $30 \mu \mathrm{m}$ \\
\hline Outer frame height & $h_{\mathrm{dpm}}$ & $1200 \mu \mathrm{m}$ \\
\hline Outer frame width & $w_{\mathrm{dpm}}$ & $1300 \mu \mathrm{m}$ \\
\hline Inner frame height & $h_{\mathrm{spm}}$ & $840 \mu \mathrm{m}$ \\
\hline Inner frame width & $w_{\mathrm{spm}}$ & $940 \mu \mathrm{m}$ \\
\hline Drive sub-suspension beam height & $h_{1}$ & $190 \mu \mathrm{m}$ \\
\hline Drive main suspension beam height & $h_{2}$ & $260 \mu \mathrm{m}$ \\
\hline Drive suspension beam width & $w_{1}$ & $6 \mu \mathrm{m}$ \\
\hline Anchor size & $w_{2} \times h_{3}$ & $40 \mu \mathrm{m} \times 40 \mu \mathrm{m}$ \\
\hline Number of drive comb frames & & 8 \\
\hline Drive comb frame height & $h_{5}$ & $200 \mu \mathrm{m}$ \\
\hline Drive comb frame width & $w_{3}$ & $25 \mu \mathrm{m}$ \\
\hline Number of comb fingers in a drive comb frame & & 15 \\
\hline Drive comb finger size & $w_{4} \times h_{4}$ & $50 \mu \mathrm{m} \times 3 \mu \mathrm{m}$ \\
\hline Drive comb finger gap & & $2.5 \mu \mathrm{m}$ \\
\hline Gap between two comb fingers in the same frame & $g$ & $8 \mu \mathrm{m}$ \\
\hline Drive finger overlap length & $l_{\mathrm{dfo}}$ & $10 \mu \mathrm{m}$ \\
\hline Ellipse 1 sense suspension beam size & $a_{1} \times b_{1}$ & $150 \mu \mathrm{m} \times 20 \mu \mathrm{m}$ \\
\hline Ellipse 2 sense suspension beam size & $a_{2} \times b_{2}$ & $144 \mu \mathrm{m} \times 14 \mu \mathrm{m}$ \\
\hline Drive mass & $m_{\mathrm{d}}$ & $0.5452 \times 10^{-7} \mathrm{Kg}$ \\
\hline Sense mass & $m_{\mathrm{s}}$ & $0.9408 \times 10^{-11} \mathrm{Kg}$ \\
\hline Drive mode stiffness & $K_{\mathrm{d}}$ & $347 \mathrm{~N} / \mathrm{m}$ \\
\hline Sense mode stiffness & $K_{\mathrm{s}}$ & $540 \mathrm{~N} / \mathrm{m}$ \\
\hline
\end{tabular}


Table 2. Several Oscillation Modes of Proposed Gyroscope

\begin{tabular}{lc}
\multicolumn{1}{c}{ Oscillation modes } & Frequency $(\mathrm{Hz})$ \\
\hline First (driving mode) & 13544.7 \\
Second & 62268.7 \\
Third & 106921.8 \\
Fourth & 140865.5 \\
\hline
\end{tabular}

This paper introduces a differential suspension structure which allows two driving proof-masses to create mechanic differential displacement along the $x$-axis. The suspension structure is shown in figure 5 and the designed parameters are listed in the table 3 . The eight anchors constrain structure to move along $y$-axis only. The $x$-axis stiffness is $121 \mathrm{~N} / \mathrm{m}$ and the $y$-axis stiffness is $2314 \mathrm{~N} / \mathrm{m}$.

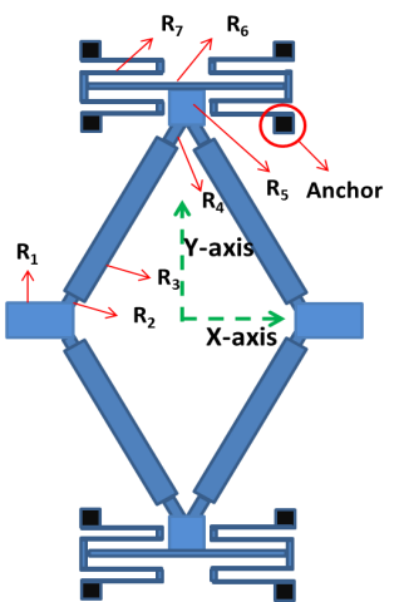

Figure 5. The structure of Differential Driving Suspension Coupling Spring

Table 3. The Designed Parameters of the Differential Driving Suspension Coupling Spring

\begin{tabular}{ll}
\hline \multicolumn{1}{c}{ Parameter } & \multicolumn{1}{c}{ Value } \\
\hline$R_{1}$ & Width $\times$ Height: $132 \mu \mathrm{m} \times 100 \mu \mathrm{m}$ \\
$R_{2}$ & Width $\times$ Height: $70 \mu \mathrm{m} \times 20 \mu \mathrm{m}$ \\
$R_{3}$ & Width $\times$ Height: $60 \mu \mathrm{m} \times 600 \mu \mathrm{m}$ \\
$R_{4}$ & Width $\times$ Height: $60 \mu \mathrm{m} \times 20 \mu \mathrm{m}$ \\
$R_{5}$ & Width $\times$ Height: $60 \mu \mathrm{m} \times 80 \mu \mathrm{m}$ \\
$R_{6}$ & Width $\times$ Height: $500 \mu \mathrm{m} \times 6 \mu \mathrm{m}$ \\
$R_{7}$ & Width $\times$ Height: $200 \mu \mathrm{m} \times 6 \mu \mathrm{m}$ \\
Total size & Width $\times$ Height: $1000 \mu \mathrm{m} \times 1700 \mu \mathrm{m}$ \\
Rotation angle of $R_{3}$ & 30 degree \\
Anchor size & Width $\times$ Height: $40 \mu \mathrm{m} \times 40 \mu \mathrm{m}$ \\
Structure thickness & $30 \mu \mathrm{m}$ \\
$x$-axis stiffness & $121 \mathrm{~N} / \mathrm{m}$ \\
$y$-axis stiffness & $2314 \mathrm{~N} / \mathrm{m}$ \\
\hline
\end{tabular}

Two single gyroscopes are linked by the differential driving suspension coupling spring to form proposed tuning fork gyroscope (see figure 6). This design constrains the two top and bottom corners A and $\mathrm{B}$ moving along $y$-direction only thanks to anchors. The two rest corners $\mathrm{C}$ and $\mathrm{D}$ are connected to the two driving frames. When having excited driving signals with the same amplitude but phase reversal put in two single gyroscopes, two driving frames will also move with the same amplitude but phase reversal. Thanks to the differential driving suspension coupling spring, the oscillation of two driving frames is always compensated each other. If appearing excited driving signals are phase mismatch (non-phase reversal), the differential driving suspension coupling spring will compensate the driving oscillation like the electronic differential amplifier. Therefore, the mismatched oscillation components are eliminated and the matched ones are amplified. In the special case, the excited signal is only applied in the left or the right driving frame of the gyroscope, the TFG still works normally due to the differential driving suspension coupling spring suspension girder structure. This proposed structure will deliver driving oscillation to both the driving frames 
thanks to its symmetrical design. However, oscillation amplitude of the two driving frames of the gyroscope is smaller when both two driving frames are excited.

In this the proposed TFG, the drive mode stiffness of whole system $K_{d T F G}=1700 \mathrm{~N} / \mathrm{m}$. The outermost device area is $4000 \mu \mathrm{m} \times 1900 \mu \mathrm{m}$ with $30 \mu \mathrm{m}$ thick device layer. Materials used in this design and simulation are polysilicon and air. Where, the TFG is made by polysilicon and surrounding by air.

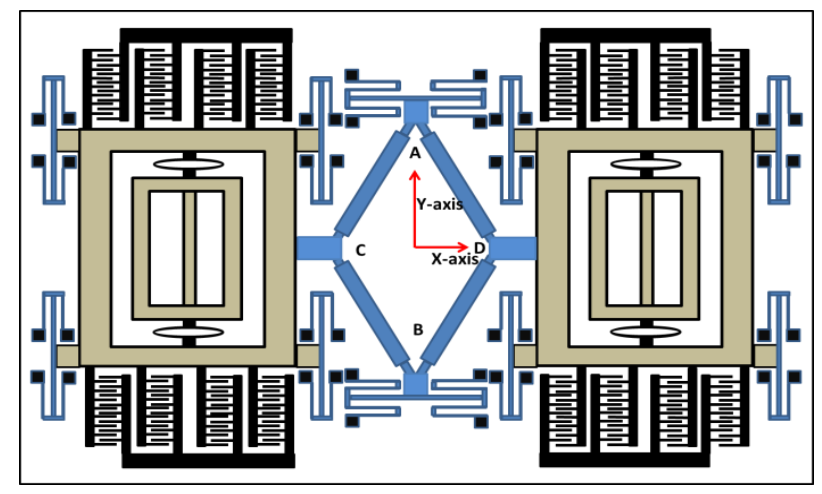

Figure 6. Design of the Proposed Tuning Fork Gyroscope. Two Driving Frame are Connected Together by a Differential Driving Suspension Coupling Spring

As mentioned in section 2.2, the proposed TFG improves the sensitivity and much rejects the common-mode noise from both driving and sensing sections. Hence, the particular purpose of this study is to demonstrate that the two single gyroscopes correspond to the two BJT common emitter amplifiers and the differential driving suspension coupling spring corresponds to the constant current source in the electronic differential amplifier. The diamond coupling spring can compensate the differential phase of two driving excited signals. It means that when two excited signals applied into driving comb frames of two gyroscopes are differential to a certain value, the differential phase of mechanical oscillation of two driving frames is still constant. The common vibration mode between the two driving frames is ignored when the differential vibration mode is much amplified.

Figure 7 shows a SEM picture of fabricated devices based on SOI substrate and Deep-RIE silicon etching with one mask fabrication process.

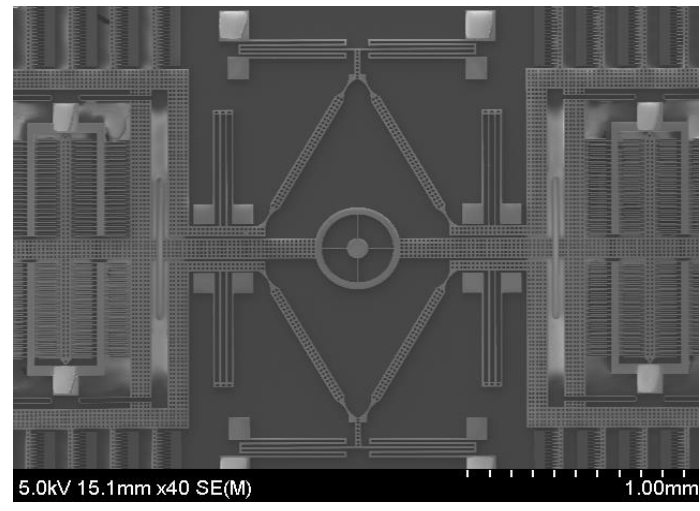

Figure 7. A SEM Picture of Fabricated Gyroscope

\section{SIMULATION}

Whole designed and simulation processes are implemented in a finite element modeling software COMSOL MULTIPHYSICS 4.4 (COMSOL Inc.). This software can be used in a lot of application areas such as Microelectromechanical systems (MEMS); Structural mechanics; heat transfer; Microfluidics etc. Physics interfaces in COMSOL allow performing various types of studies including: stationary and 
time-dependent (transient) studies; linear and nonlinear studies; eigenfrequency, modal, and frequency response studies.

Materials utilized in this work are polysilicon and air. Excited signals can be force $(N)$ or voltage potentials $(V)$ with various kinds of waveform such as sinusoidal, triangular, trapezoid. However, in order to implement and simulate faster and simpler, instead of voltage potentials, a force function is directly applied to the driving frame. Eigenfrequencies; stiffness; displacement of single gyroscope, differential driving suspension coupling springs and the TFG are then simply extracted from simulation results.

\section{RESULTS AND DISCUSSION}

Before demonstrating that the proposed TFG has differential phase compensation ability between two input excited signals, it needs to build some TFG architectures corresponding to various stiffness of the differential driving suspension coupling spring. The main purpose of building various architectures is to find a certain differential phase value of two input excited driving signals that the proposed TFG can compensate.

However, in order to obtain maximum displacements of drive proof-mass and sense proof-mass, it needs to use the most suitable frequency in the excited signal equations. In this case, it is the driving resonant frequency of the TFG. This frequency can be found via finite element method in Comsol Multiphysics software version 4.4 (Study/Study steps/Eigenfrequency/Eigenfrequency).

Some finite element analysis results of the single gyroscope achieved by COMSOL are shown in Figure 8. Eigenfrequencies are shown in Table 2.

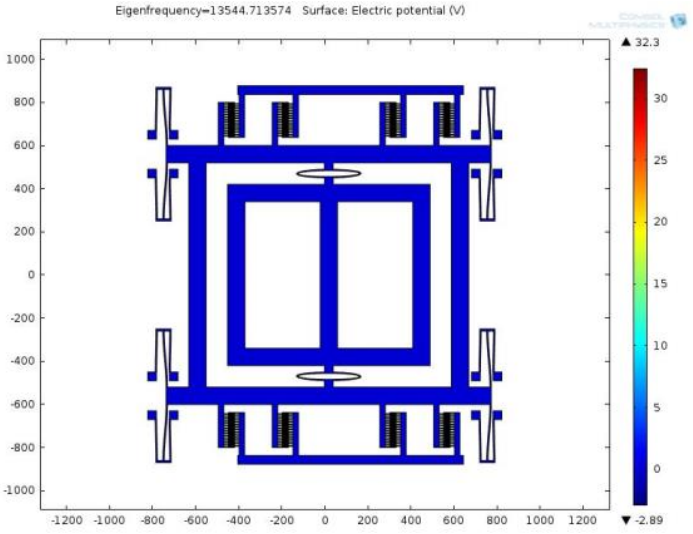

(a)

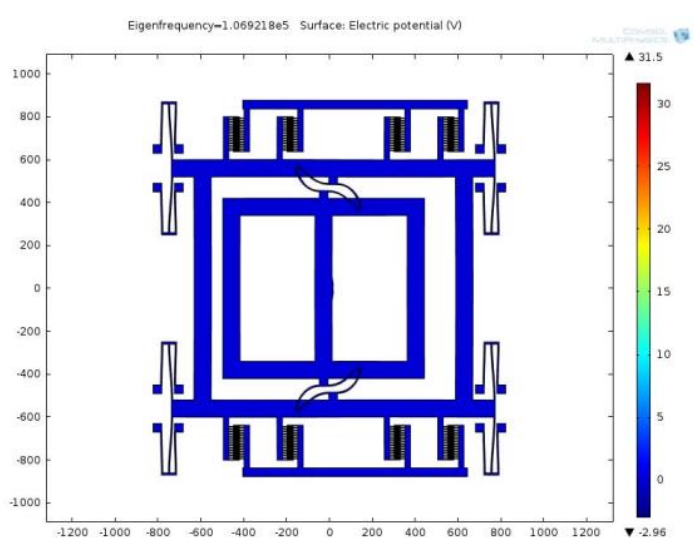

(c)

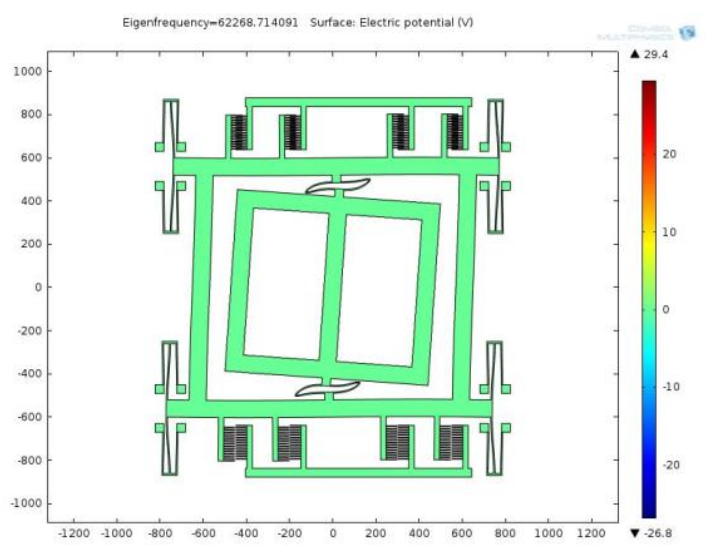

(b)

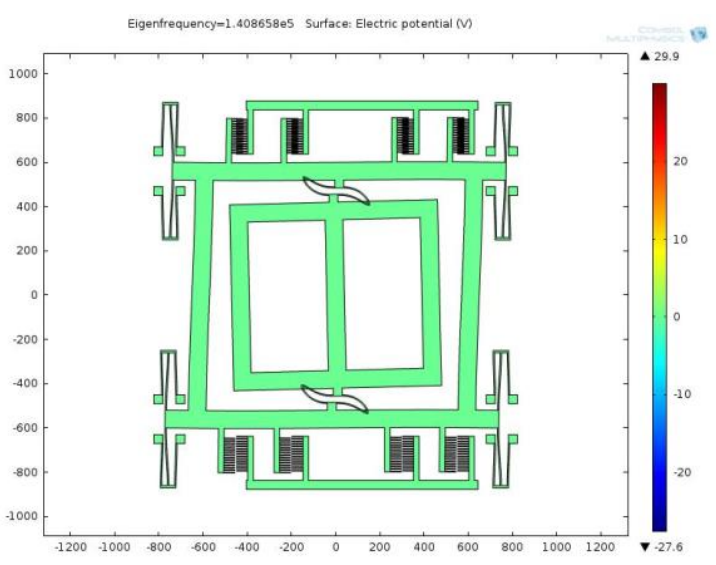

(d)

Figure 8. The Finite Element Analysis Result of Gyroscope Obtained by COMSOL: Driving Mode (a); Some other Modes (b, c, d) 
In this simulation, the stiffness of the differential driving suspension coupling spring between two gyroscopes is changed to get the optimal value. Specifically, the heights of the coupling suspension beam $R_{6}$ in Figure 8 of structure 1, 2, 3 are $6 \mu \mathrm{m}, 4 \mu \mathrm{m}, 12 \mu \mathrm{m}$, respectively while the rest parameters of structures are unchanged.

Some finite element analysis results of the first proposed TFG (structure 1) achieved by COMSOL are shown in Figure 9 (the similar way is implemented with the second and the third TFGs). Some oscillation modes of three structures are shown in the Table 4.

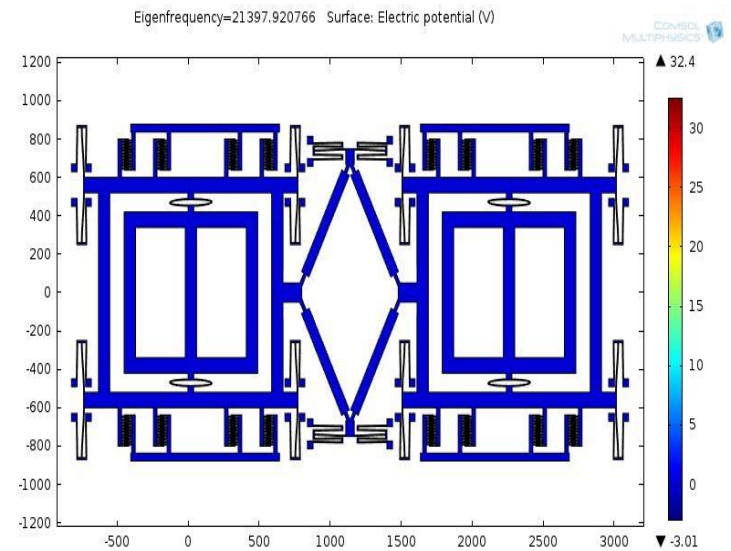

(a)

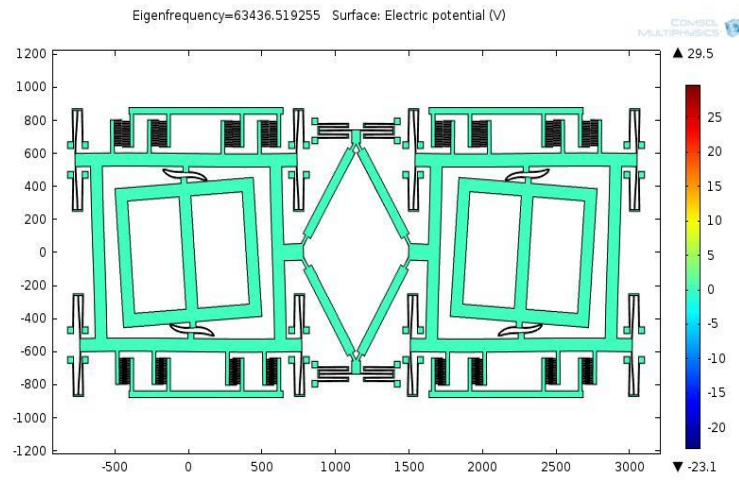

(c)

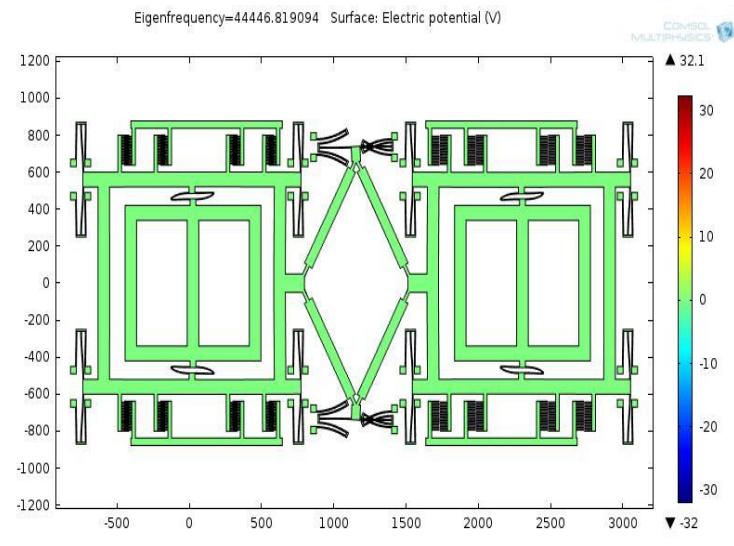

(b)

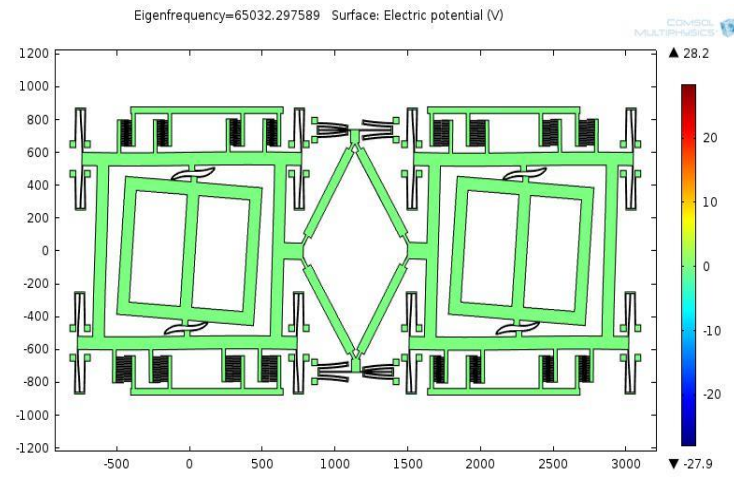

(d)

Figure 9. The Finite Element Analysis Result of TFG Structure 1 Obtained by COMSOL: Driving Mode (a); other Modes (b, c, d)

Table 4. Lowest Driving Related Oscillation Modes of Three TFG Structures

\begin{tabular}{cccc}
\hline Modes & \multicolumn{3}{c}{ Frequency $(\mathrm{Hz})$} \\
Structure 1 & Strure 2 & Structure 3 \\
\hline First (Driving mode) & 21397.9 & 21184.7 & 21751.2 \\
Second & 44446.8 & 44227.6 & 44801.3 \\
Third & 63436.5 & 63427.9 & 63447.1 \\
Fourth & 65032.2 & 65020.9 & 65049.6 \\
\hline
\end{tabular}

The parameters in the table 4 show that the stiffer the differential driving suspension coupling spring is, the higher the driving consonant frequency of TFG is and vice versa. Driving resonant frequency of the first structure is shown in Figure 10. During the simulating process, the structures have the same mesh settings: sequence type is physics-controlled mesh and element size is extremely coarse. The structure is designed by polysilicon material and assumed to be immersed in air. 
The two driving excitation signals are applied to the driving combs having formulas:

$$
\begin{aligned}
& f_{1}=\sin (2 * p i * f * t) \mathrm{N} \\
& f_{2}=\sin (2 * p i * f * t+\varphi) \mathrm{N}
\end{aligned}
$$

where $f_{l}$ signal is put in eight driving comb frames of gyroscope on the left and $f_{2}$ signal is put in eight driving comb frames of gyroscope on the right.

The process of simulating results is individually performed for each above structure. So, $f$ frequency in Equation 1 and Equation 2 is the driving eigenfrequency (the driving resonant frequency) correspondent to each structure. For example, $f=21397.9 \mathrm{~Hz}$ is applied to structure 1 . In the conventional working principle of the tuning fork gyroscope, the $f_{l}$ and $f_{2}$ two excited signals are anti-phase. It means that $\varphi=180$ degree.

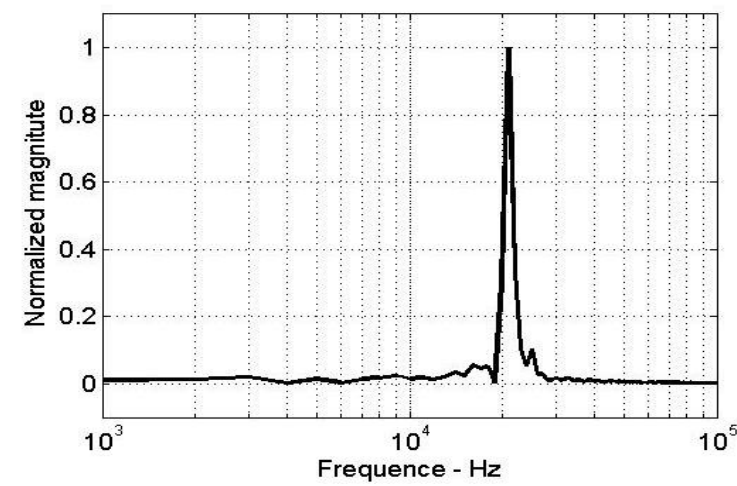

Figure 10. Driving Resonant Frequency of the Proposed Architecture 1

However, in this study the excitation signal $f_{1}$ is kept stable while $f_{2}$ is changed with various $\varphi$ phase: $180^{\circ}, 179.5^{\circ}, 179^{\circ}, 178.5^{\circ}, 178^{\circ}$ etc. in a computing time of a specific structure.

The aim of changing $\varphi$ in $f_{2}$ is to observe mechanical vibration differential phase of two driving proof-masses in order to determine differential phase compensation ability of the proposed TFGs when having the differential phase between two input excitation signals.

Figure 11 shows an $x$-axis simulated displacement profile of the first TFG (structure 1) at the time of 5e-4 s (Figure 11a) and 5.12e-4 s (Figure 11b). It indicates that the mechanical vibrations of the two driving frames are well matched.

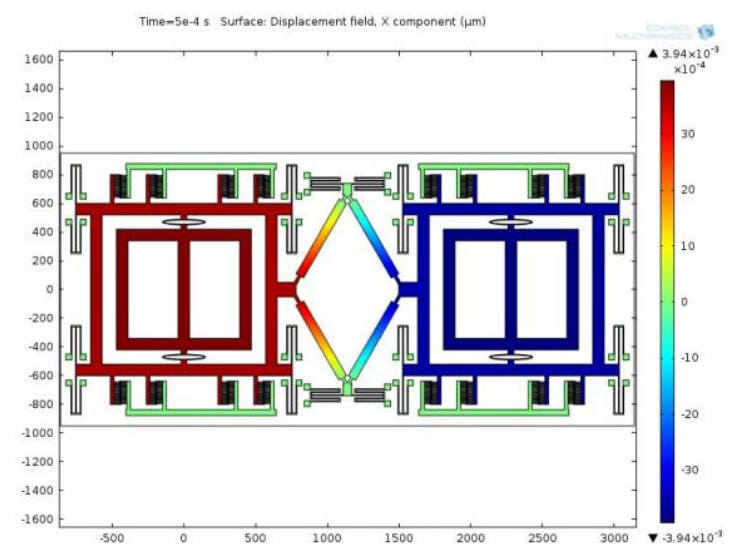

(a)

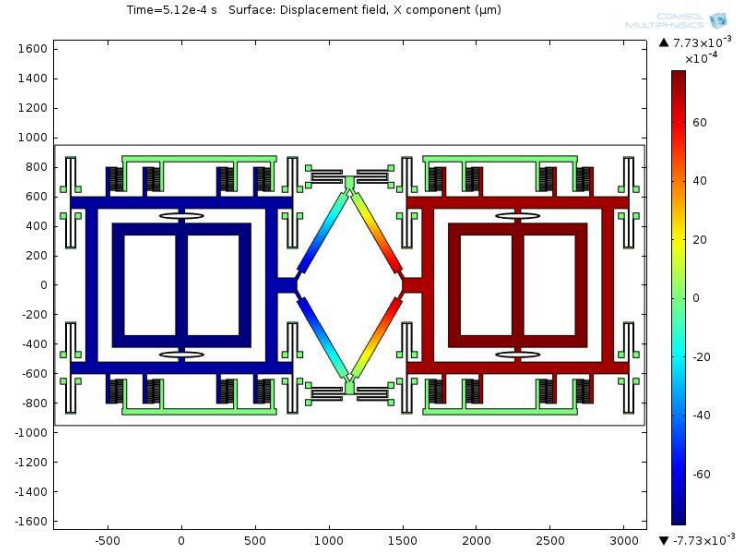

(b)

Figure 11. Simulated Displacement Profile of the Proposed TFG 
Table 5 shows the relation between input excited signal differential phase $\varphi$ and mechanical displacement signal differential phase $\varphi_{1}$ in three structures. These results are drawn in Figure 12.

Table 5. Relation between $\varphi$ and $\varphi_{1}$

\begin{tabular}{cccc}
\hline$\varphi$ & Structure 1 & Structure 2 & Structure 3 \\
\hline $180^{\circ} / 0^{\circ}$ & 0 & 0 & 0 \\
$179.5^{\circ} / 0.5^{\circ}$ & 0 & 0 & 0 \\
$179^{\circ} / 1^{\circ}$ & 0 & 0 & 0 \\
$178.5^{\circ} / 1.5^{\circ}$ & 0 & 0 & 0 \\
$178^{\circ} / 2^{\circ}$ & 0 & 0 & 0 \\
$177.5^{\circ} / 2.5^{\circ}$ & 0 & 0 & 0 \\
$177^{\circ} / 3^{\circ}$ & 0 & 3.5 & 0 \\
$176.5^{\circ} / 3.5^{\circ}$ & 0 & 7.0 & 0 \\
$176^{\circ} / 4^{\circ}$ & 3.9 & 15 & 0 \\
$175.5^{\circ} / 4.5^{\circ}$ & 18.2 & $\mathrm{x}$ & 15.6 \\
\hline Unit: Degree & & &
\end{tabular}

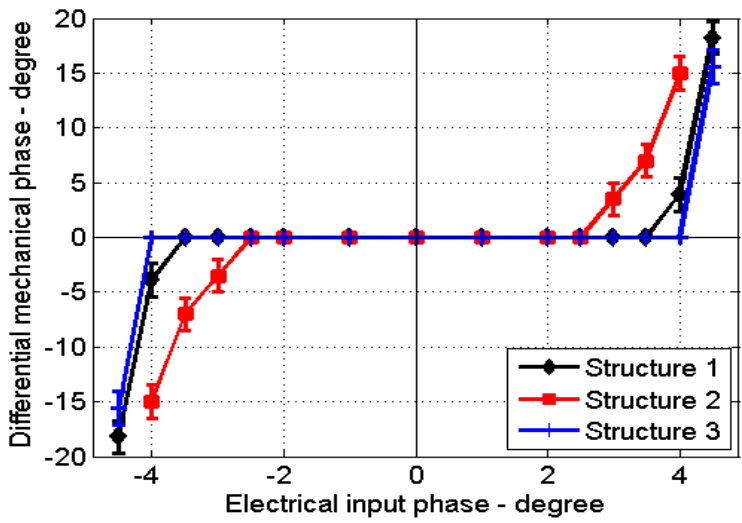

Figure 12. Mechanical Vibration Differential Phase between Two Driving Frames Versus Electrical Driving Phase Difference

Above results shows that the mechanical vibration is well matched when the driving excitation phase different is less than $3.5^{\circ}, 2.5^{\circ}$ and $4^{\circ}$ corresponding to structure 1,2 and 3 , respectively with driving signal of $1 \mathrm{~N}$ amplitude and $0 \mathrm{~N}$ offset. The mechanical vibrations of two driving frames are not compensated for the excited driving differential phase more than $3.5^{\circ}, 2.5^{\circ}$ and $4^{\circ}$ corresponding to structure 1,2 and 3 . In this case, the mechanical vibration is fully dominated by the excited signal. Basing on the results of the three structures, the structure 3 is the best one thanks to the wide range of the excited driving differential phase up to $4^{\circ}$. However, the resonant frequency of this structure is also the highest (see Table 4).

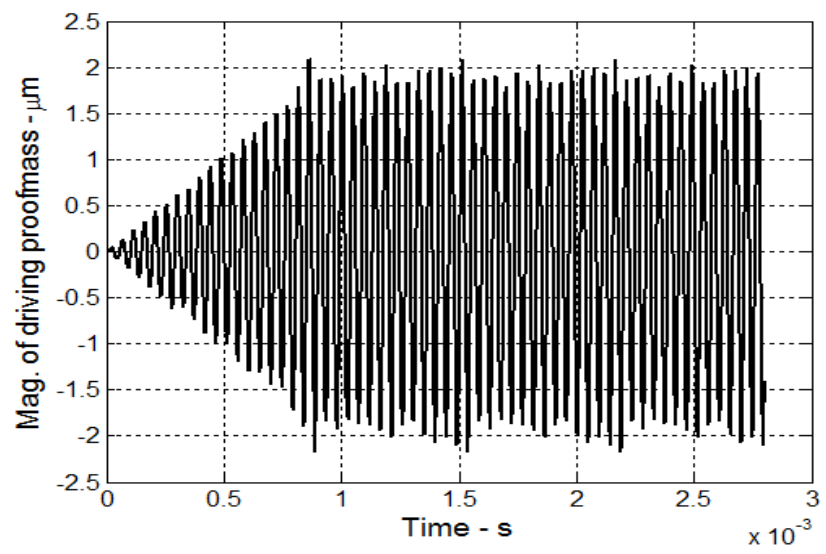

Figure 13. Driving Proof-Mass Displacement of the Proposed TFG 
Figure 13 points out the displaced magnitude and signal shape of driving proof-mass of the left gyroscope of the proposed TFG (the displacement of driving proof-mass of the right gyroscope has the same magnitude and is anti-phase with the left one - not shown here). In the case of structure 1 , the results show that in the period of $2.8 \times 10^{-3} \mathrm{~s}$, oscillation amplitude gradually increases from 0 to about $1.9 \mu \mathrm{m}$ (from 0 to about $0.9 \times 10^{-3} \mathrm{~s}$ ). After this period, the structure is approached stable state. The oscillation amplitude is about $1.9 \mu \mathrm{m}$.

In order to determine the displaced magnitude of sensing proof-mass, in this study, the angular rate $\omega$ is changed by sinusoidal signal (Figure 14a); triangular signal (Figure 15a) and trapezoid signal, respectively (Figure 16a) while the driving excited signals are still constant as shown in Equation 1 and Equation 2 (using structure 1). The oscillation amplitudes of sensing proof-mass corresponding to the above signals are shown in Figure 14b, Figure 15b and Figure 16b, respectively.

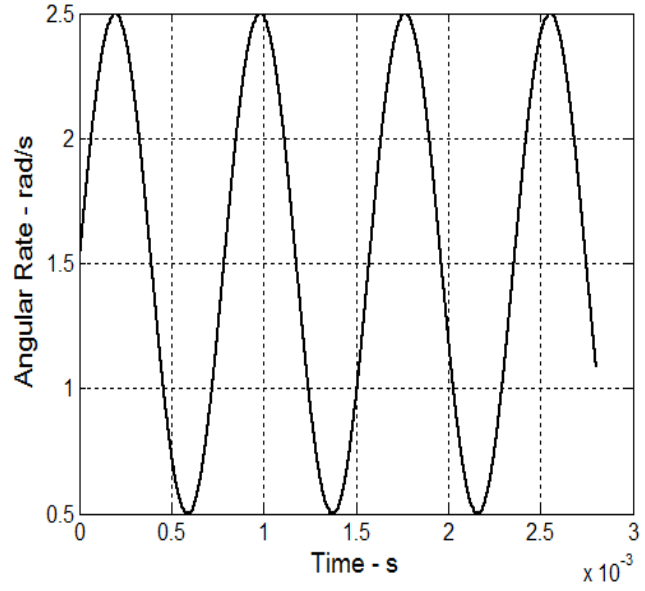

(a)

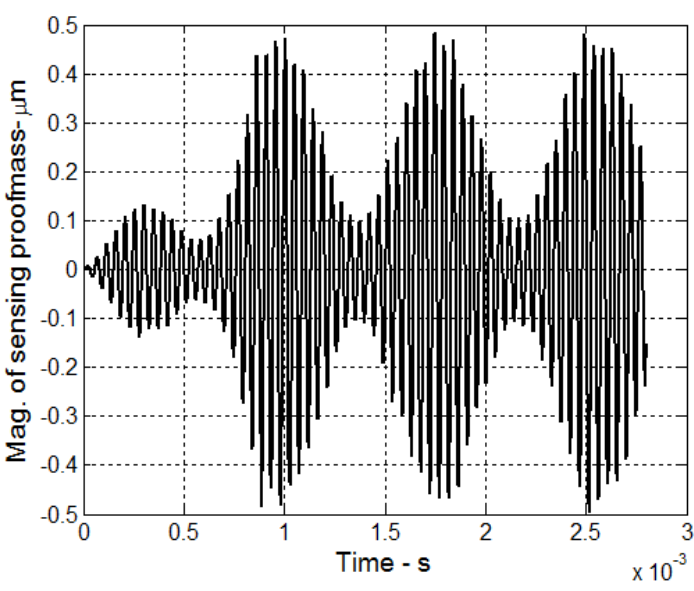

(b)

Figure 14. Mechanical Displacement of Sensing Proof-Mass (b) when Having Sinusoidal Angular Rate (a)

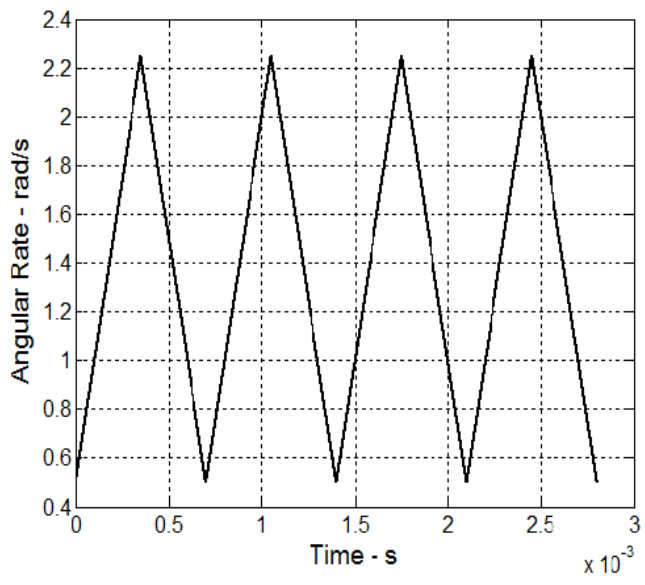

(a)

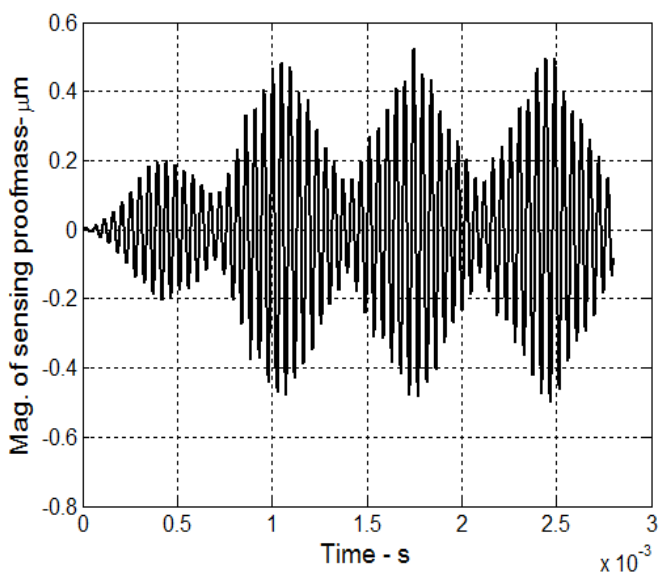

(b)

Figure 15. Mechanical Displacement Of Sensing Proof-Mass (b) when Having Triangular Angular Rate (a)

The simulation results in Figure 13 to Figure 16 show responses of the proposed TFG with a rather highly stable oscillation amplitudes of drive proof-mass and sense proof-mass. Figure 17 shows the measured output voltage versus input angular rate of the proposed tuning fork gyroscope. The sensor linearly operates in the range from -200 to +200 degree/s with output voltage of $-0.09 \mathrm{~V}$ to $+0.09 \mathrm{~V}$, respectively. The sensor resolution is about $0.45 \mathrm{mV} / \mathrm{degree} / \mathrm{s}$. The further measurement of the fabricated sensor will be implemented for investigating the mechanical, electrical properties, and time response. 


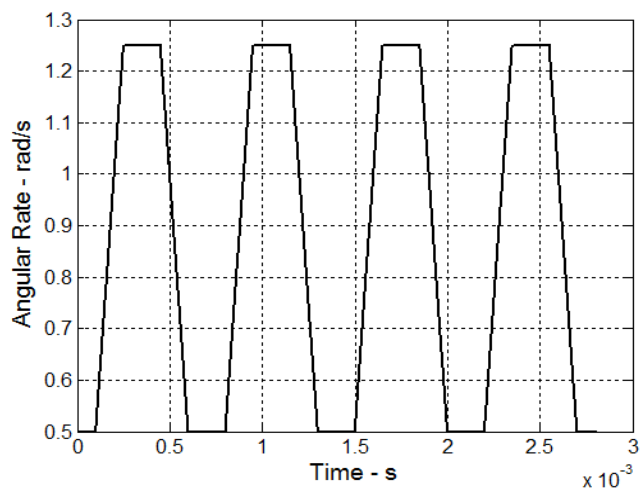

(a)

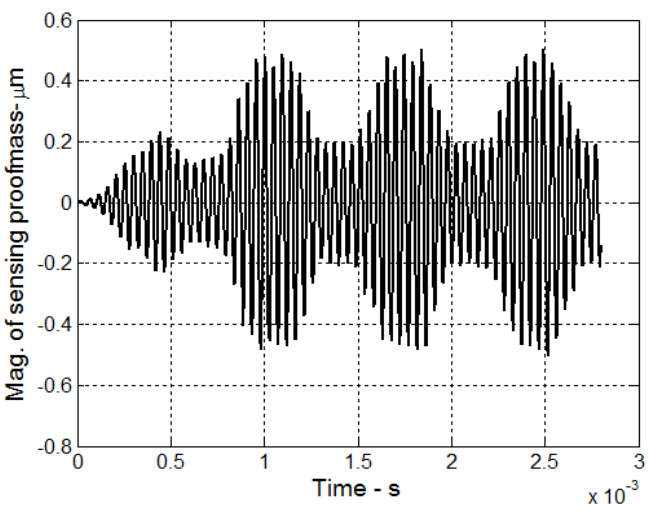

(b)

Figure 16. Mechanical displacement of sensing proof-mass (b) when having trapezoid angular rate (a)

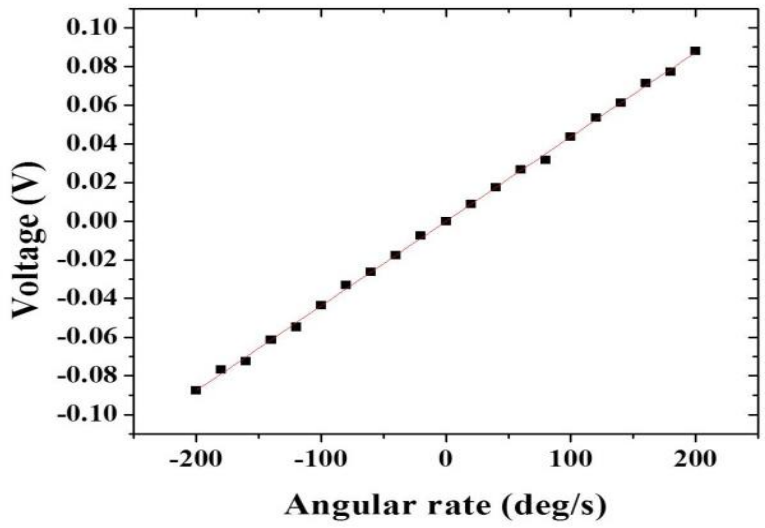

Figure 17. Output Voltage Versus Input Angular Rate

\section{CONCLUSION}

A novel tuning fork gyroscope with a differential suspension structure between two driving frames are designed, fabricated and characterized. The driving frame mechanical vibration is well matched when the electrical driving differential phase is less than $3.5^{\circ}$ in structure $1 ; 2.5^{\circ}$ in structure $2 ; 4^{\circ}$ in structure 3 . The differential gained parameters are, therefore, much improved when the common vibration modes between the two driving frames are ignored. The fabricated tuning fork gyroscope linearly operates in the range from -200 to +200 degree $/ \mathrm{s}$ with resolution of about $0.45 \mathrm{mV} /$ degree/s.

\section{ACKNOWLEDGEMENTS}

This work is partly supported by NAFOSTED project coded 103.99-2014.34. The authors would like to thank T. Bui Duc of the Faculty of Electronics and Telecommunications for the support on simulation setup.

\section{REFERENCES}

[1] IEEE Std 528-2001, IEEE Standard for Inertial Sensor Terminology.

[2] Ayazi, Farrokh, and Khalil Najafi. "Design and fabrication of high-performance polysilicon vibrating ring gyroscope”, Micro Electro Mechanical Systems, 1998. MEMS 98. Proceedings, the eleventh annual international workshop on. IEEE, 1998.

[3] Maenaka, Kazusuke, et al, "Design, fabrication and operation of MEMS gimbal gyroscope", Sensors and Actuators A: Physical, 121.1 (2005): 6-15.

[4] Yazdi, Navid, Farrokh Ayazi, and Khalil Najafi. "Micromachined inertial sensors", Proceedings of the IEEE 86.8 (1998): 1640-1659. 
[5] Sang Won Yoon, Sangwoo Lee, Khalil Najafi. "Vibration-induced errors in MEMS tuning fork gyroscopes", Sensors and Actuators A: Physical, (2012): 32-44.

[6] Weinberg, Marc S., and Anthony Kourepenis. "Error sources in in-plane silicon tuning-fork MEMS gyroscopes", Journal of Microelectromechanical Systems, 15.3 (2006): 479-491.

[7] Liu, Guangjun, et al, "Effects of environmental temperature on the performance of a micromachined gyroscope", Microsystem Technologies, 14.2 (2008): 199-204.

[8] Cenk Acar, Andrei Shkel,MEMS Vibratory Gyroscopes: Structural Approaches to Improve Robustness, (2008). p. 8 section, "1.5 Applications of MEMS Gyroscopes".

[9] Andrei M. Shkel. "Type I and Type II Micromachined Vibratory Gyroscopes", in Proc. IEEE/ION Position Locat. Navigat. Symp., San Diego, CA, Apr. 24-27, 2006: 586-593.

[10] D. Piyabongkarn, R. Rajamani, M. Greminger. "The development of a MEMS gyroscope for absolute angle measurement", IEEE Transactions on Control Systems Technology, 13 (2005): 185-195.

[11] K. Liu, W. Zhang, W. Chen, K. Li, Fu. Dai, F. Cui, X. Wu, G. Ma, Q. Xiao. "The development of micro-gyroscope technology", Journal of Micromechanics and Microengineering, vol 19, no 11 (2009).

[12] M.W. Putty. "A micromachined vibrating ring gyroscope", Ph.D dissertation, Univ. Michigan, Ann Arbor, MI, 1995.

[13] K. Shcheglov. "DRG-A hight performance MEMS gyro", in Proc. Joint Precision Azimuth Sensing Symp., Las Vegas, NV, Aug. 2-4, 2010.

[14] Alexander A. Trusovs, Adam R.Schofield, Andrei M. Shkel. "Micromachined rate gyrocope architecture with ultrahigh quality factor and improved mode ordering", Sensors and Actuators A: Physical, 165 (2010): 26-34.

[15] Alexander A. Trusovs, Igor P. Prikhodko, Sergei A. Zotov, Andrei M. Shkel. "Low-Dissipation Silicon Tuning Fork Gyroscopes for Rate and Whole Angle Measurements", IEEE Sensors Journal, vol. 11, no. 11, November 2011: 2763-2770.

[16] Sheng-Ren Chiu, Chung-Yang Sue, Chih-Hsiou Lin, Yan-Kuin Su. "Dual-axis tuning fork vibratory gyroscope with anti-phase mode vibration mechanism", Microsystem Technology, (2014): 2173-2184.

[17] Hung Ngoc Vu, Dzung Viet Dao, Hoang Manh Chu, Thong Quang Trinh, Long Quang Nguyen, and Trinh Chu Duc. "Design and analysis of a lateral axis tuning fork gyroscope with guided-mechanical coupling", International workshop on soft and flexible micro/nano systems technology (2013), Australia.

[18] Simon, Brenton R., Alexander A. Trusov, and Andrei M. Shkel."Anti-phase mode isolation in tuning-fork MEMS using a lever coupling design", Sensors, 2012 IEEE. IEEE, 2012.

[19] Simon, Brenton R., Alexander A. Trusov, and Andrei M. Shkel."Lever mechanisms for anti-phase mode isolation in MEMS tuning-fork structures", U.S. Patent No. 9,217,756. 22 Dec. 2015.

[20] Adel. S. Sedra, Kenneth C. Smith. "Microelectronic circuit", Oxford University Press, $6^{\text {th }}$ edition, 2009.

\section{BIOGRAPHIES OF AUTHORS}
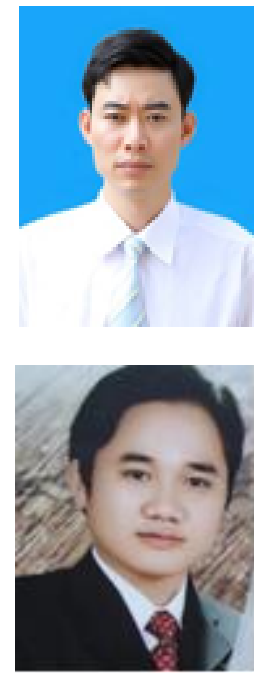

Thang Nguyen Van was born in 1979. He received his B.Sc., degree in Electronics and Telecommunication at the Hanoi University of Transport and Communications, Hanoi, Vietnam, in 2002 and his M.Sc. degree in Information Engineering from Le Quy Don University, Hanoi, Vietnam, in 2007. He has been a lecturer of Broadcasting College I under Radio the voice of Vietnam since 2003. He becomes vice leader of training department of Broadcasting College I since 2007. Now, he is PhD student of the University of Engineering and Technology (UET), Vietnam National University Hanoi, Vietnam (VNUH). Until now, He is author and coauthor of some papers on MEMS based sensors and their application.

Email: nguyenbathangvov@gmail.com

Tran-Duc Tan was born in 1980. He received his B.Sc, M.Sc, and PhD. degrees respectively in 2002, 2005, and 2010 at the University of Engineering and Technology (UET), Vietnam National University - Hanoi, Vietnam (VNUH), where he has been a lecturer since 2006. He was the recipient of the Vietnam National University, Hanoi, Vietnam Young Scientific Award in 2008. He is currently an Associate Professor with the Faculty of Electronics and Telecommunications, University of Engineering and Technology, Vietnam National University, Hanoi, Vietnam. He is author and coauthor of 30 papers on MEMS based sensors and their application. His present research interest is in DSP applications.

Email: tantd@vnu.edu.vn 

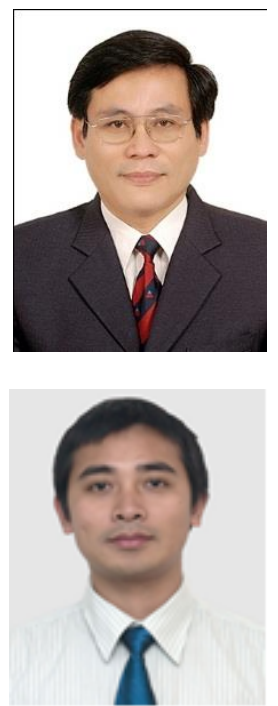

Hung Vu Ngoc is an Associate Professor of Materials Science at Hanoi University of Science and Technology (HUST), Vietnam. He received Bachelor degree in Physics from Kishinev University, Russia (1979) and PhD in Physics from HUST in 1991. Currently, he is the leader of MEMS Lab. at International Training Institute for Materials Science (ITIMS). His research interests range from ferroelectric materials to MEMS technology applied for inertial MEMS devices.

Email: hungvungoc@itims.edu.vn

Trinh Chu Duc received the B.S. degree in physics from Hanoi University of Science, Hanoi, Vietnam, in 1998, the M.Sc. degree in electrical engineering from Vietnam National University, Hanoi, in 2002, and the Ph.D. degree from Delft University of Technology, Delft, The Netherlands, in 2007. His doctoral research concerned piezoresistive sensors, polymeric actuators, sensing microgrippers for microparticle handling, and microsystems technology. He is currently an Associate Professor with the Faculty of Electronics and Telecommunications, University of Engineering and Technology, Vietnam National University, Hanoi, Vietnam. Since 2008, he has been the Vice-Dean of the Faculty of Electronics and Telecommunications. He has been chair of Microelectromechanical Systems and Microsystems Department, since 2011. He has authored or coauthored more than 50 journal and conference papers. He was the recipient of the Vietnam National University, Hanoi, Vietnam Young Scientific Award in 2010, the 20th anniversary of DIMES, Delft University of Technology, The Netherlands Best Poster Award in 2007 and the 17th European Workshop on Micromechanics Best Poster Award in 2006. He is guest editor of the Special Issue of "Microelectromechanical systems" Vietnam journal of Mechanics, in 2012.

Email: trinhcd@vnu.edu.vn 